\title{
Women's Experiences in the Antenatal and Perinatal Period Following a Stillbirth or Neonatal Death: A Multicentre Cohort Study
}

\section{Suzanne Thomas}

Saint Mary's Hospital, Manchester University NHS Foundation Trust, Oxford Road, Manchester, M13 9WL.

\section{Louise Stephens}

Saint Mary's Hospital, Manchester University NHS Foundation Trust, Oxford Road, Manchester, M13 9WL.

\section{Tracey A Mills}

Liverpool School of Tropical Medicine

\section{Christine Hughes}

Saint Mary's Hospital, Manchester University NHS Foundation Trust, Oxford Road, Manchester, M13 9WL.

\section{Alan Kerby}

Maternal and Fetal Health Research Centre, Division of Developmental Biology and Medicine, Faculty of Biology, Medicine and Health, University of Manchester, UK

\section{A. Michael Arundale}

Alliance Manchester Business School, The University of Manchester, Booth St West, Manchester, M15 6PB

\section{Debbie M Smith}

Manchester Centre for Health Psychology, School of Health Sciences, Faculty of Biology, Medicine and Health, University of Manchester

Alexander E. P. Heazell ( $\square$ alexander.heazell@manchester.ac.uk)

Maternal and Fetal Health Research Centre, Division of Developmental Biology and Medicine, Faculty of Biology, Medicine and Health, University of Manchester, UK

\section{Research Article}

Keywords: Perinatal Death, Stillbirth, Neonatal Death, Subsequent Pregnancy, Pregnancy after loss, Anxiety, Depression, Perceived Stress, Dual Process Model of Bereavement, Mixed methods.

Posted Date: March 30th, 2021

DOl: https://doi.org/10.21203/rs.3.rs-345719/v1 
License: (c) (i) This work is licensed under a Creative Commons Attribution 4.0 International License. Read Full License 


\section{Abstract \\ Background}

The death of a baby before or shortly after birth is a profoundly distressing experience for women and their families. Although grieving the death of their baby, most women will embark on another pregnancy. Specialist antenatal services have been proposed to address the increased biomedical and psychological risks in pregnancies after perinatal death. This study aimed to explore the experiences of women in and shortly after pregnancy after loss to determine the psychological and economic impact and the effect of a specialist service.

\section{Methods}

This study adopted a mixed-methods approach using several sources of data: i) validated measures of psychological state (Cambridge Worry Score, Edinburgh Postnatal Depression Score (EPDS), Generalized Anxiety Disorder 7-item score), ii) measurement of hair cortisol, iii) face-to-face semi-structured interviews to explore women's views and experiences of care during their pregnancy, and iv) a Social Return on Investment (SROI) analysis with a subgroup of women and staff participants at the lead site.

\section{Results}

In total 112 women participated in the study. Measures of anxiety and depressive symptoms decreased from the highest levels at 15 weeks' gestation to 6-weeks postnatal (for example mean GAD-7: 15 weeks $8.2 \pm 5.5,6$ weeks postnatal $4.4 \pm 5.0, p<0.001$ ). Hair cortisol levels fell in a similar profile to anxiety and depression symptoms $(p<0.05)$. Thematic analysis of interviews $(n=20)$ described how stillbirth was a quiet, unspoken subject and that navigating subsequent pregnancies relied on expecting the worst and hoping for the best, mapping these themes onto the Dual Process Model of Bereavement found being pregnant complicated the grieving process as increased awareness of the risk of stillbirth drew parents focus back to loss. Attendance at a specialist service was valued; SROI analysis found that for $£ 1$ invested, $£ 6.10$ of value was generated, mostly relating to reduced negative psychological symptoms.

\section{Conclusions}

This mixed-methods study demonstrated heightened anxiety and depressive symptoms and elevated cortisol levels which decrease as pregnancy progresses. Specialist care was viewed favourably, and is cost-effective, but comparative studies are required to determine whether this model is superior to routine high-risk care and to identify which components are most valued.

\section{Background}


In the UK, approximately 1 in 250 babies are stillborn and 1 in 600 babies die in the first month of life; thus, 4,100 families are bereaved each year [1]. The death of a baby before or shortly after birth (hereafter referred to as perinatal death) is a profoundly distressing experience for women and their families and is invariably followed by a period of grief [2]. Although grieving, women frequently report planning for another pregnancy [3], and most will embark on another pregnancy, with estimates of $50 \%$ of women conceiving within a year and $86 \%$ within 18 months $[4,5]$. Previous perinatal death is consistently recognised to increase parents' anxiety, perceived stress, emotional vulnerability and decrease their confidence in outcome of the next pregnancy $[6,7]$. This is a cause for concern because elevated maternal anxiety increases the risk of adverse pregnancy outcomes, notably preterm birth and low birthweight [8]. Furthermore, a longitudinal study reported that the negative psychological impacts of perinatal death persist beyond the next pregnancy despite the birth of a healthy child [9]; previous history of perinatal death has been reported to disrupt maternal attachment and negatively impact on parenting [10].

In a subsequent pregnancy, many mothers and fathers report the loss of 'normal' positive feelings they expected and have described how their subsequent pregnancies were characterised by heightened anxiety and fear [7]. These emotions are compounded by common societal misconceptions, such as the new pregnancy helps parents to 'get over' grief for a dead child which isolates parents from social support networks, increasing their reliance on professionals or other parents with similar experiences. Qualitative studies of women's experiences of subsequent pregnancies highlight the value placed on regular interaction with health professionals $[11,12]$. This suggests that specialist antenatal support might ameliorate anxiety, improve experiences of pregnancy, support relationships and positively impact on future parenthood [13]. To address the need for increased support and specialist care in subsequent pregnancies, dedicated "pregnancy after loss" services have developed. An evaluation of 10 women attending such a clinic in a tertiary maternity unit in Australia found seven themes from semi-structured interviews relating to respondent's experiences of the pregnancy after loss clinic and other services and recommendations for improvement [14]. This study recommended further evaluation of such antenatal services with larger, representative samples, which include assessment of quantitative outcomes such as maternal or paternal mood, and relationship with the baby [14].

A specialist pregnancy after loss service (the Rainbow Clinic) was established at Saint Mary's Hospital, a tertiary maternity unit in Manchester, UK in 2014. This was expanded to a neighbouring maternity unit in Wythenshawe, South Manchester in 2016. A The model of care provided by the clinic has been described in detail elsewhere [15], but focuses on continuity of care provided by an experienced multidisciplinary team consisting of obstetricians, midwives, bereavement midwives and administrative staff with access to specialist perinatal bereavement counselling if required. Care follows the international consensus statement for care in pregnancies after stillbirth [16] and is individualised based upon prior history of loss, maternal medical disorders and findings on ultrasound scans performed throughout the pregnancy. This study aimed to explore pregnancy and postnatal experiences of women in pregnancies after perinatal death and assess the economic value of the specialist service at the two sites delivering this model of care. 


\section{Methods}

Following ethical approval (Ref 16/NW/0258) the study was conducted between 22/09/2016 and 21/12/2018 at three sites: St Mary's Hospital and Wythenshawe Hospital, Manchester and Royal Preston Hospital, UK. At the first two sites there was a specialist service (Rainbow Clinic) for care after perinatal death and at the latter there was a dedicated bereavement midwife to support families following the death of their baby.

Pregnant women were eligible for inclusion if they were attending the antenatal service for care in a pregnancy after perinatal death. Women were excluded if they were less than 16 years of age, lacked capacity to consent or who had been diagnosed with pregnancy complications or received treatment for an acute mental health issue in this pregnancy. Staff who worked in the clinic were asked to participate in the focus groups.

The study employed a mixed-methods design using several sources of data: i) validated measures of psychosocial factors, ii) a sample of hair was obtained in a subgroup of women at the last clinic appointment for measurement of cortisol and iii) face-to-face semi-structured interviews conducted with a sub-group of women to explore their views and experiences of care during their pregnancy. and iv) social return on investment analysis undertaken at the two sites with a specialist clinic incorporating interviews with patient and staff participants. To reduce the burden, women participated in either the face to face qualitative interviews or the social return on investment analysis but not both.

\section{Completion of Validated Questionnaires}

Participants were asked to complete the Cambridge Worry Score [17], Edinburgh Postnatal Depression Score [18] and Generalised Anxiety and Depression 7-item score [19]) at three time-points: approximately 15 weeks' gestation, 32 weeks' gestation and 6 weeks postpartum. Responses were scored at the completion of the study and entered into a study database. The proportion of GAD-7 scores > 10 were taken to indicate significant anxiety [19] and EPDS > 13 were taken to indicate significant depressive symptoms [20].

\section{Hair Cortisol Measurements}

A $0.5 \mathrm{~cm}$ diameter of hair at the vertex of the head was cut at the scalp and stored at $-20^{\circ} \mathrm{C}$. The hair was trimmed down to $13 \mathrm{~cm}$ from the scalp end and washed in high purity isopropanol on a rotator for 3 minutes. After being air-dried at room temperature for 2 days, it was cut into consecutive $3 \mathrm{~cm}$ samples representing hair growth from each trimester. $60 \mathrm{mg}$ of hair was minced with a scalpel and ground in a bead beater for 5 minutes (Bullet Blender, Next Advance). Ground hair samples were incubated in $1.5 \mathrm{ml}$ of high purity methanol for 18 hours at room temperature on a rotator. After being centrifuged at 10,000 rpm for 5 minutes $1 \mathrm{~mL}$ of the supernatant was transferred to a tube and dried in a vacuum evaporator (MiVac, Genevac). The extract was re-suspended in $250 \mu \mathrm{l}$ phosphate buffered saline. Samples were assayed in duplicate using the standard protocol from a cortisol ELISA kit (ALPCO) and measured at 450 
$\mathrm{nm}$ in a spectrophotometer (Omega, BMG Labtech). Duplicate measurements with a coefficient of variation greater than $40 \%$ were excluded from the analysis. Sample cortisol measurements were normalised for sample hair weight.

\section{Statistical analysis for Quantitative Data}

Quantitative data were entered into Microsoft Excel for descriptive statistical analysis. Comparative analysis was undertaken in STATA Version 14 (StataCorp, TX, USA) to determine whether there were differences in responses to psychometric questionnaires at different stages of pregnancy. Distribution of data was evaluated by Shapiro-Wilk test. Normally distributed data were analysed by one-way ANOVA and non-normally distributed data were analysed by Kruskal-Wallis test, matched data were analysed by repeated measures ANOVA or Friedman test depending on whether the data were normally distributed. Due to an absence of preliminary data in this population a formal sample size calculation was not performed to inform study size. A p value of $<0.05$ was taken to indicate statistical significance.

\section{Semi-structured interviews and qualitative analysis}

The interview topic guide was designed by research team with input from a patient panel who had attended the research clinics at St Mary's Hospital. It comprised four sections (history leading to care pathway, their experience of coping with new pregnancy after loss, support and advice for others). Two interviews were planned, one in the antenatal period and one in the postnatal period. Telephone interviews were conducted by one of two researchers (postgraduate students) with training and support from the research team digitally audio-recorded and transcribed verbatim by the researcher.

Two steps to analysing the qualitative data were undertaken; the focus of the analysis was on the current pregnancy and the experience of health care in this pregnancy. The six-stages of thematic analysis as described by Braun and Clarke (2006) were used as a template to analyse qualitative data from the interviews and identify semantic level themes [21]. DS, a psychologist with expertise of research methodologies but no personal experience of stillbirth or neonatal death and who had not been involved in conducting the interviews, conducted the initial stages of the thematic analysis (stage 1 to 4 ) and then the experiences of $\mathrm{AH}$ and ST were introduced at stage 5 and 6 to allow for theme discussion and contextualisation.

The second step of analysis was undertaken once the themes had been constructed through the steps described above. The aim of this step was to understand the women's current pregnancy experience with the lens of an established model of bereavement to help further explain their care needs. A deductive approach was taken and one researcher (DS) mapped the themes and subthemes onto the Dual Process Model of Coping with Bereavement (Stroebe \& Schut, 1999 [22]) and through discussions with AH and ST some suggested edits were made to the model to fully encompass the experiences of women pregnant after previous losses.

\section{Social Return on Investment Analysis}


A Social Return on Investment (SROI) analysis attempts to capture the intangible, hard to measure social and environmental value of an intervention in financial units, ie $£$ of value delivered per $£$ spent. As the intangible costs of stillbirth are considerable [23], and are likely larger than direct costs, a SROI methodology was employed to evaluate the impact of Rainbow Clinic on the lead site [24]. The SROI analysis employed a six-stage approach: i) establishing scope and identification of stakeholders, ii) mapping outcomes via focus groups with patients, partners and staff, iii) evidencing the outcomes and giving them a value, iv) establishing the impact and the degree to which this was attributable to the Rainbow Clinic, v) calculating the SROI and vi) reporting and embedding the information. In total, 18 women, 3 partners, 4 Rainbow Clinic staff, 2 stakeholders from other maternity units and one representative each from St Mary's Hospital and Tommy's charity contributed to determining the scope of the analysis and relevant stakeholders. Two focus groups of 21 participants and a service user survey (n $=14$ ) were used to measure outcomes and correct for deadweight, attribution and displacement. Indicators and proxy variables were identification from currently available secondary data including NHS reference costs. The SROI was reported as the ratio of the value generated by the clinic and the costs of providing the service. Sensitivity analyses were conducted to determine the effect of changing input costs and the value of outputs.

\section{Results}

Participation in the elements of the study is shown in Fig. 1. The 112 participants were between 21 and 46 years of age at enrolment to the study (Table 1). The majority of women $(70.5 \%)$ were of White British ethnicity; the sample included women from other White groups, Black African, South Asian and other ethnic groups. The largest group of women were married (45.5\%), although a large proportion described themselves as single (26.8\%). The majority of women were in paid employment $(63.4 \%)$, although a large proportion of women did not report this information (24.1\%). Participation in individual elements of the study varied, 87 women completed the questionnaire element of the study, 13 gave hair samples, 24 women had interviews and 35 women participated in the SROI analysis (some participants completed more than one component of the study). Of the 87 women who completed the initial questionnaire at 15 weeks' gestation, 62-65 completed the questionnaires at 32 weeks' gestation and 52-54 completed the questionnaires 6 weeks postnatal (Fig. 1). The reasons for not completing subsequent questionnaires included pregnancy loss, giving birth before 32 weeks' gestation and loss to follow-up. As some scores were missing individual components between 44-46 participants completed all three questionnaires. 
Table 1

- Demographic characteristics of study participants $(n=112)$.

\begin{tabular}{|l|l|}
\hline Characteristic & Median (range) for continuous data \\
\hline N (\%) for categorical data
\end{tabular}

\section{Quantitative data}

Cambridge Worry Score (CWS) demonstrated a reduction from the highest levels at 15 weeks' gestation to lower levels at 6 weeks postnatal (Fig. 2A), when analysis was restricted to participants who returned 
all three questionnaires $(n=46)$ there was a stepwise reduction between the three time points studied (Fig. 2B). The profile of GAD-7 scores similarly found with the lowest levels seen at 6 weeks postnatal, but there was no difference in GAD-7 scores between 15 and 32 weeks' gestation (Fig. $2 \mathrm{C}$ and D). Applying a threshold for significant anxiety of a GAD score $\geq 10$ found $38 \%$ of participants had significant anxiety at 15 weeks' gestation, $27 \%$ at 32 weeks' gestation and $23 \%$ at 6 weeks postnatal. Edinburgh Postnatal Depression Score (EPDS) was again lowest at 6 weeks postnatal compared to during pregnancy, however, there was no reduction between scores at 15 and 32 weeks' gestation (Fig. 2E and F). Applying a threshold value $>13$ in the EPDS found $30.1 \%$ had significant depressive symptoms at 15 weeks' gestation which fell to $27.7 \%$ at 32 weeks' gestation and $11.1 \%$ postnatally. The profile of GAD-7 and EPDS scores was the same when analysis was restricted to participants who returned all questionnaires

(Fig. 2D and F). The strongest correlation was seen between GAD-7 and EPDS scores $\left(r^{2}=0.65\right)$, with weaker relationships between GAD-7 and CWS $\left(r^{2}=0.19\right)$ and EPDS and CWS $\left(r^{2}=0.17\right)$. The responses to the visual analogue score about quality of life suggested higher scores at 6 weeks postnatal, which was statistically significant when analysis was restricted to women with complete questionnaire responses (Fig. $2 \mathrm{G}$ and $\mathrm{H}$ ). There was a negative relationship between EPDS scores and reported quality of life $\left(r^{2}=0.12\right)$. Hair cortisol levels were reduced by $48 \%$ in the third trimester compared to the first trimester (Fig. 3).

\section{Qualitative study}

Twenty women were interviewed during pregnancy with six of these being interviewed for a second time after birth. The antenatal interviews had a mean length of 38 minutes and the postnatal interviews had mean duration of 25 minutes. Of these 20 women, 13 reported one previous stillbirth, three reported two stillbirths, three reported one neonatal death and one reported both a neonatal death and stillbirth. The number of children that women had given birth to ranged from one to eight.

In the inductive analysis two themes were identified in the antenatal interviews to encapsulate the women's experiences of their current pregnancy following previous loss(es): 'It's just such a quiet and unspoken subject' and 'Expect the worst, hope for the best'. Both themes included two subthemes each which are outlined below with excerpts from the interview transcripts to provide context. Expressed emotions are described in the words used by the women in the interviews so are in italics.

\section{Theme 1: 'Its just such a quiet, unspoken subject' (Alice)}

The women's increased awareness of the risks in pregnancy due to their previous loss(es) of a baby influenced their experience in this pregnancy and as one woman called it ' $m y$ reality' (Hazel). Several of the women had experienced the death of more than one baby. Women reflected that as the death of a baby was not openly talked about in society they previously had low awareness. The resulting shock and guilt felt following their previous loss(es) carried through into this pregnancy and the women talked about the ways they protected themselves and their baby to attempt to create a state of normality. Their experience in subsequent pregnancies was noted to be different 'I don't really feel like a normal expectant 
parent'(Sarah). Two subthemes described navigating their subsequent pregnancy: protective environment of clinic and a need to protect others.

Protective environment of clinic: Attending the Rainbow Clinic in this pregnancy was described by a number of women as reassuring as it offered a protected environment. Women felt protected in several ways. Firstly, they felt relief at not having to repeat their pregnancy story at each appointment and the negative emotions associated with talking through the details with different health professionals. Secondly, they felt they could trust the staff as they were being cared for by the same team of health professionals who had time for them and did not rush their appointment. It was acknowledged by all women that the Rainbow Clinic staff were experts, who could provide personal care to them, the link to the stillbirth research programme also increased confidence in the team. Being cared for by the same health professionals throughout their pregnancy was emphasised by a number of women as something that felt familiar and reduced stress. Thirdly, they felt they were not alone as they knew the other women at the clinic were in the same situation as them and they could relate to them. This also made them feel a '...sense of validation...' (Jessie) as their situation was recognised by the staff and peers. However, this also felt daunting to some women due to an awareness of the sadness felt by all of the patients. Finally, the increased frequency of the appointments and the flexibility to arrange additional appointments if desired was valued by the women as it made them feel more relaxed. Being under the care of the Rainbow Clinic and in this protected environment led women to feel less anxiety and was referred to after birth as a '...security blanket...' (Cassie).

'...Since I joined Rainbow Clinic, my anxiety has been less, erm definitely...they've really taken care of my stress in every possible way' (Maya)

'It feels like with the Rainbow Clinic, the anxiety is cut out before it even has a chance to exist. It's just more personalised care...' (Hope)

'It feels like we're protected and we've realised that we are different, but it's like our own personal space...' (Chloe).

After birth, women expressed general disappointment with postnatal care after hospital discharge and many highlighted a lack of any postnatal follow up from the specialist clinic. The necessity of focus on antenatal monitoring and support in subsequent pregnancy was recognised, but the lack of contact in the postnatal period left women feeling abandoned.

'... I feel like you have built up a closer relationship...it was a bit weird never hearing or seeing them again after that...' (Sarah).

Some of the women talked about the birth of their baby and milestones after this, reminding them of what they did not have with the child that they lost and they reported a negative impact on their wellbeing. 
A need to protect others: The women acknowledged that their awareness of baby loss was not shared by others and they frequently mentioned wanting to protect other people who did not have this experience. They felt it was their responsibility to protect other people, especially partners, family and friends, from the negative emotions that they experienced.

'It's not just me that's worried...it's that ripple effect of this one thing affecting so many other people as well' (Geordie Mama)

Women talked about their partners having little support and finding it difficult to express their feelings and the impact of these on their mental wellbeing '...men don't speak about emotions really, he's just like one of these everything will be fine and if it's not, he's one of these that deals with it when it does happen' (Natalia). To protect close friends and family, women delayed telling them about their current pregnancy as they feared how they would cope with the new situation. Likewise, women avoided mixing with other pregnant women (such as standard antenatal classes) as they did not want to share experiences and expose them to the reality of loss and did not want them to feel uncomfortable if asked about their pregnancy story. Finally, some women wanted to protect the child they lost as they did not want their current pregnancy or baby to replace them, this led to feels of guilt,

'I just felt guilty every time I got pregnant like I was betraying him, kinda thing'(Natalie).

\section{Theme 2: 'Expect the worst, hope for the best' (Alice)}

As stated above, women were more aware of the risks due to their previous experiences and entered their current pregnancies with trepidation. This increased awareness of risks led to heightened levels of anxiety and fear at certain points in pregnancy including the period immediately before scans or appointments and the end of the pregnancy. Women felt responsible for their baby and felt mixed emotions about their pregnancy as a result of the fear of another loss. Many spoke of the desire to stop being led by fear and to stop feeling angry as this was disruptive to their wellbeing, to do this women engaged two approaches which are summarised in these subthemes: Control and hope.

I feel bad about it because like I'm so grateful to be in this position, but yeah I feel so bad because I'm so scared and so worried and upset' (Lauren)

'I want to move on and move forward' (Geordie Mamma)

Control: Women felt responsible for their baby and they felt they had little control over their previous loss(es) so they attempted to exert control over their emotions and their behaviours in their current pregnancy. A few women outlined that they did not let themselves form a psychological and physical attachment with their baby, seeing them not give the baby a name or buy any items for their baby. The Rainbow Clinic offered them control as they were given more appointments and scans when they needed them which offered reassurance. 
'...all the reassurance that you do get has been fabulous. Knowing that if I need extra scans, if I need to come in and speak to someone, all I have to do is ring up...' (Julia)

'I can't let myself get excited about it because you know, I don't know you almost become disassociated with it because I think because you don't really want to let yourself get too excited about it' (Michelle).

Hope: Women wanted to have hope as they wanted to enjoy the pregnancy and this baby, however, this was challenged by their increased awareness of risk. Women expressed a mixture of feelings as their pregnancies progressed, some women felt more anxiety as they reached the gestation of their previous loss(es) and one mentioned feeling like they were '..walking a tight rope everyday..' (Judith) until the end of pregnancy. Whereas, some felt more confident and hopeful in their pregnancy outcome. These mixed feelings in pregnancy saw many women supress feelings of excitement for the current pregnancy. Feeling movements of their baby for many women felt reassuring and gave them hope and the absence of movement was something that women reported as being anxiety provoking during the early stages of the pregnancy. When discussing partner's feelings about hope, women outlined similar attitudes expressed by their partners.

'...the first part you are anxious but...you don't know what's happening inside you... rely on the baby's movements...' (Sophia)

'he's [partner] warming up now but he's always waiting for something to go wrong' (Julia).

These mixed feelings led to many women not preparing psychologically and physically for their baby. The postnatal interviews saw participants reflect that they have not accepted what it would be like if they gave birth to a live baby which they took home with them. A few women reported seeking support from healthcare providers and also charities to support their postnatal preparation, but the rest of the women did not have any support and felt this was needed to enable them to both prepare psychologically and manage their emotions. Several women reported feeling anxious and panic about how to care for their baby as they had not allowed themselves to feel emotional attachment to a baby and had not attended any antenatal education groups.

'...it was very, very difficult bringing him home and S [husband] and I cried a lot on the way home because we just got upset that we hadn't had the opportunity to bring J home...' (Geordie Mama)

'...it [speaking to the counsellor] was more about talking through the emotions of what it would meant to hopefully bring home a, you know a baby, a health baby at the end of this given that had happened last time, how that was going to affect me, how it was going to bring things back...' (Sarah)

Using a deductive approach, the themes and subthemes were mapped onto the Dual Process Model of Coping with Bereavement according to womens' expressed emotions and experiences of coping during their subsequent pregnancies. Since their loss, the women had been coping with their bereavement through their 'everyday life experiences'. However, during these interviews the women were experiencing their bereavement during pregnancy, the life experience that was directly related to their loss and 
bereavement, this complicated the oscillation process between loss-orientation and restorationorientation. Furthermore, they had an increased awareness of the risk of pregnancy loss which drew focus back to loss. This added element to the model sees women reliving the psychological and physiological state of pregnancy and all the time-specific events associated with this such as scans and baby movements (Fig. 4).

The emotions associated with theme 1 (It's such a quiet unspoken subject) of shock and guilt were associated with the loss-orientation state. Whereas, the coping strategies used by the women across both themes (four subthemes) were located in the restoration-orientation. Theme one (expect the worst, hope the best) showed the journey of women from the loss-orientation state to the restoration-orientation state.

\section{Social Return on Investment Analysis}

The focus group discussions and interviews with parents, staff and stakeholders identified 14 outcomes of the clinic for quantification. The patient-related outcomes were: reduced healthcare costs, reduced experience of depression and anxiety during pregnancy, greater connection with baby, reduced isolation and a greater sense of control and ability to plan and reduced post-natal depression. The proportion of change related to attendance at the specialist clinic ranged from $64 \%$ for reduced postnatal depression through to $100 \%$ for reduced use of ultrasound or attendance with other health professionals (Fig. 5A). The social return on investment was calculated to be $£ 1,358,400$ compared to the cost of running the service of $£ 223,958$, giving a ratio of 6.10 (i.e. for every $£ 1$ invested $£ 6.10$ of benefit was derived). This was dominated by the value attached to the birth of a live baby $(£ 499,944)$, if this value were subtracted, the total social return on investment was $£ 858,456$ giving a ratio of 3.81 (Fig. $5 B$ ).

\section{Discussion}

This mixed methods study provides a holistic view of women's experiences in the antenatal and immediate postnatal period in a pregnancy following a stillbirth. It also describes the impact of attending a specialist antenatal service by reducing participant's psychological symptoms and consequent health behaviours e.g. attending additional appointments. The Social Return on Investment analysis suggested that a specialist antenatal service provides significant value and better outcomes for families and the NHS without increasing costs.

\section{Strengths and Limitations}

This study is strengthened by the combination of quantitative measures of maternal anxiety and depression which have been validated for use in pregnancy, qualitative interviews and social return on investment methodology to capture the intangible costs of pregnancy after stillbirth. However, this study did not employ a comparative design so conclusions cannot be drawn about the levels of anxiety or depression in comparison to pregnant women without a history of stillbirth. As not all women participated there may be a selection bias which could impact upon the responses. Similarly, not all women completed all three questionnaires, in some cases this was because women had given birth 
before 32 weeks' gestation, and in other cases because a mother didn't participate or moved from one of the participating units. In both cases, it is plausible that non-participants may have had higher levels of anxiety or depressive symptoms which would underestimate the impact of prior stillbirth on these symptoms in a subsequent pregnancy.

\section{Contextualising the findings}

Although there is a wealth of evidence that pregnancy after loss requires additional antenatal care in terms of support from professionals and investigations to identify recurrent or related conditions to help parents navigate the increased risk of psychological and medical complications [7], there are few studies that evaluate the impact of specialist antenatal services [16]. Warland and O'Leary identified that "support and early intervention at the time of stillbirth and subsequent pregnancy is likely to be useful. Further research is needed to determine whether early intervention can alter the tendency to paradoxical parenting style [10]." The importance of research into care in pregnancies after loss was emphasised by its inclusion into stillbirth research priorities identified by both parents and professionals [25].

The findings presented here provide a comprehensive view of the experiences of women attending a specialist antenatal service for perinatal loss. Whilst this study was carried out in three sites in the NorthWest of the UK the findings are similar to those reported elsewhere, indicating that the findings are likely generalisable. Hughes and Turton reported average EPDS scores of 10.2 in the third trimester of pregnancy after stillbirth and $28 \%$ scored "high", which was comparable to 9.2 and $28 \%$ in our sample respectively [26]. Our findings of increased symptoms of anxiety and depression are also in agreement with other earlier reviews describing symptoms in pregnancy after loss [27]. Interestingly, our data suggest that the levels of symptoms of anxiety and depression are highest at 15 weeks' gestation and then fall as pregnancy progresses. This change could reflect increased belief that the pregnancy will result in a healthy outcome, or that contact with specialist antenatal service reduced the incidence of these symptoms; both these possibilities were reported in the qualitative data.

During the study we also introduced measurement of hair cortisol as evidence emerged that this may reflect prenatal stress and relates to progression of depressive symptoms [28,29]. The values of hair cortisol showed wide variation, but were in keeping with previously reported levels [28] and showed the same downward pattern as seen in the psychometric questionnaires. Interestingly, this is the opposite pattern to a study describing hair cortisol levels in normal pregnancy [30]. This method is evolving, and there is a need to standardise collection, storage and measurement techniques so that results can be compared between studies [28]. Nevertheless, this appears to be an objective means by which prenatal stress could be measured in women in pregnancy/ies after stillbirth but further work is required to determine whether the values obtained in this population differ from women without a history of loss.

The themes derived from the qualitative interviews showed similarities with several of the seven themes identified by Meredith et al. in their thematic analysis of ten parents attending a pregnancy after loss clinic (PALC) in Brisbane, particularly with components of the unique experience of pregnancy after loss and support from the PALC [14]. When reviewing the overall experience of pregnancy after loss in both

Page 14/ 24 
studies, women describe the mixture of emotions highlighted by other studies and also of guilt for having another child, while not wanting to forget their stillborn baby. In both studies women viewed a specialist service positively, giving security, understanding and reassurance that their emotional responses were normal. Meredith also noted the important effects on the wider family unit which were not assessed here [14]. Further research is needed to investigate whether a specialist antenatal service for pregnancy after loss improves partner's and other family member's experience. Likewise, more work is needed to understand the emotional and psychological experiences of parents following birth as this study showed anxiety and depression scores to be high in a number of women and the interview data demonstrates women protecting themselves in case of another loss. The impact of these experiences on parenthood and mother-child attachment are not well understood, although disorganized attachment has been described [31].

In their seminal papers on the Dual Process Model of grief, Stroebe and Schutt describe that grief is very personal and experienced in an iterative manner [22], individuals who have greater restoration oriented behaviour have better adjustment after the death of a child, these behaviours to some extent buffered the negative effects of loss oriented experiences [32]. When the themes derived from the qualitative interviews were mapped to the Dual Process Model it is clear that in pregnancy after loss that restoration oriented behaviours predominate, but that navigating pregnancy after loss parents relive events that led to the loss of their baby which leads to increased oscillation between loss and restoration oriented behaviours. This increases our theoretical understanding of the process of bereavement and provides a cogent reminder that professional or lay support may be required to allow parents to develop more restoration-oriented behaviours. Further research is needed to determine how this can be best provided in pregnancies after loss and to understand how being in the same physiological state with physical reminders (e.g., scan appointments for pregnancy) can influence the bereavement process

Pregnancies after loss are associated with increased resource use [33], Hutti et al. described that resource use was associated with increased maternal anxiety and depressive symptoms [34]. One potential reason for this is that women whose care needs are not being met, may have increased anxiety and depression and also seek additional appointments from healthcare professionals. As many costs associated with stillbirth are "intangible" [23], we adopted a SROI analysis. In agreement with the hypothesis outlined above, our study demonstrated attendance at the specialist antenatal service reduced anxiety and depressive symptoms and reduced the number of consultations during pregnancy. After the birth of a live baby, these were the largest areas of benefit derived from the specialist clinic. Our analysis suggests that the specialist clinic provides a significant return on investment in keeping with other reported interventions in reproductive health (range 1.73-21.20) [35]. Thus, wider implementation of a specialist antenatal service would be viewed as providing social value and would address current efforts to personalise maternity care (e.g. UK Maternity Transformation Programme).

\section{Conclusions}


This mixed-methods study provides a detailed view of women's experience in pregnancies after perinatal death. The methods consistently demonstrate heightened anxiety, stress and depressive symptoms which decrease as pregnancy progresses. Provision of specialist care in a dedicated clinical service was viewed favourably, but comparative studies are required to determine whether this model is superior to routine high-risk care and to identify which components of the dedicated service are valued. A recent prioritisation study found $73 \%$ of respondents indicated that this was an urgent and important research question to be addressed [36]. Further studies are also needed to understand partners' and other family member's experiences of pregnancy/ies after stillbirth to appreciate which aspects of care and support are beneficial in a future pregnancy.

\section{List Of Abbreviations}

ANOVA Analysis of Variance

CWS Cambridge Worry Score

EPDS Edinburgh Postnatal Depression Score

GAD-7 Generalized Anxiety Disorder (7-item)

PALC Pregnancy After Loss Clinic

SROI Social Return on Investment

UK United Kingdom

\section{Declarations}

\section{Ethics approval and consent to participate}

This study was given a favourable ethical opinion by Greater Manchester East Research Ethics Committee (Ref 16/NW/0258). All participants gave written informed consent prior to participation; all methods were performed in accordance with the relevant guidelines and regulations

\section{Consent for publication}

Not applicable

\section{Availability of data and materials}

The datasets generated and/or analysed during the current study are not publicly available as ethical approval was not sought for their dissemination but are available from the corresponding author on reasonable request. 


\section{Competing interests}

The authors declare that they have no competing interests

\section{Funding}

This study was funded by Tommy's charity and the Holly Martin Stillbirth Research Fund.

\section{Authors' contributions}

Contribution of Authorship: Conception (AEPH, ST, TAM, DMS), planning (AEPH, ST, TAM, DMS, AMA), carrying out (LS, ST, CH, AK), analysing (AEPH, AK, ST, DMS), writing up (AK, AEPH, ST, DMS, AMA). All authors have seen and approved the final draft of the manuscript.

\section{Acknowledgements}

We would like to thank the participating hospitals St Mary's Hospital, and Wythenshawe Hospital, Manchester and Royal Preston Hospital, Lancashire. Particularly the midwives who recruited participants: Jane Boscolo-Ryan and Katrina Rigby. Also, we would like to thank the two research students Victoria Jones and Laura Sambrook who helped with the qualitative interviews. Finally, we would like to thank all the mothers who kindly agreed to participate.

\section{References}

1. Draper ES, Gallimore ID, Kurinczuk JJ, Smith PW, Boby T, Smith LK, Manktelow BN, on behalf of the MBRRACE-UK collaboration: MBRRACE-UK Perinatal Mortality Surveillance Report, UK Perinatal Deaths for Births from January to December 2016 In. Leicester:: The Infant Mortality and Morbidity Studies, Department of Health Sciences, University of Leicester.; 2018.

2. Heazell AE, Siassakos D, Blencowe H, Burden C, Bhutta ZA, Cacciatore J, Dang N, Das J, Flenady V, Gold KJ et al: Stillbirths: economic and psychosocial consequences. Lancet 2016, 387(10018):604616.

3. Meaney S, Everard CM, Gallagher S, O'Donoghue K: Parents' concerns about future pregnancy after stillbirth: a qualitative study. Health Expect 2017, 20(4):555-562.

4. Forrest GC, Standish E, Baum JD: Support after perinatal death: a study of support and counselling after perinatal bereavement. British medical journal (Clinical research ed 1982, 285(6353):14751479.

5. Mills TA, Ricklesford C, Heazell AE, Cooke A, Lavender T: Marvellous to mediocre: findings of national survey of UK practice and provision of care in pregnancies after stillbirth or neonatal death. BMC pregnancy and childbirth 2016, 16:101.

6. Hunter A, Tussis L, MacBeth A: The presence of anxiety, depression and stress in women and their partners during pregnancies following perinatal loss: A meta-analysis. J Affect Disord 2017, 223:153164. 
7. Mills TA, Ricklesford C, Cooke A, Heazell AE, Whitworth M, Lavender T: Parents' experiences and expectations of care in pregnancy after stillbirth or neonatal death: a metasynthesis. BJOG 2014, 121(8):943-950.

8. Mulder EJ, Robles de Medina PG, Huizink AC, Van den Bergh BR, Buitelaar JK, Visser GH: Prenatal maternal stress: effects on pregnancy and the (unborn) child. Early Hum Dev 2002, 70(1-2):3-14.

9. Blackmore ER, Cote-Arsenault D, Tang W, Glover V, Evans J, Golding J, O'Connor TG: Previous prenatal loss as a predictor of perinatal depression and anxiety. Br J Psychiatry 2011, 198(5):373378.

10. Warland J, O'Leary J, McCutcheon H, Williamson V: Parenting paradox: parenting after infant loss. Midwifery 2011, 27(5):e163-169.

11. Cote-Arsenault D, Donato KL, Earl SS: Watching \& worrying: early pregnancy after loss experiences. MCN Am J Matern Child Nurs 2006, 31(6):356-363.

12. Cote-Arsenault $D$, Donato KL: Restrained expectations in late pregnancy following loss. $J$ Obstet Gynecol Neonatal Nurs 2007, 36(6):550-557.

13. Caelli K, Downie J, Letendre A: Parents' experiences of midwife-managed care following the loss of a baby in a previous pregnancy. Journal of advanced nursing 2002, 39(2):127-136.

14. Meredith P, Wilson T, Branjerdporn G, Strong J, Desha L: "Not just a normal mum": a qualitative investigation of a support service for women who are pregnant subsequent to perinatal loss. $B M C$ pregnancy and childbirth 2017, 17(1):6.

15. Heazell AE, Wojcieszek AM, Graham N, Stephens L: Care in pregnancies after stillbirth and perinatal death. International Journal of Birth and Parent Education 2018, 6(2):23-28.

16. Ladhani NNN, Fockler ME, Stephens L, Barrett JFR, Heazell AEP: No. 369-Management of Pregnancy Subsequent to Stillbirth. J Obstet Gynaecol Can 2018, 40(12):1669-1683.

17. Green JM, Kafetsios K, Statham HE, Snowdon CM: Factor structure, validity and reliability of the Cambridge Worry Scale in a pregnant population. J Health Psychol 2003, 8(6):753-764.

18. Schaper AM, Rooney BL, Kay NR, Silva PD: Use of the Edinburgh Postnatal Depression Scale to identify postpartum depression in a clinical setting. $J$ Reprod Med 1994, 39(8):620-624.

19. Spitzer RL, Kroenke K, Williams JB, Lowe B: A brief measure for assessing generalized anxiety disorder: the GAD-7. Arch Intern Med 2006, 166(10):1092-1097.

20. Cox JL, Holden JM, Sagovsky R: Detection of postnatal depression. Development of the 10-item Edinburgh Postnatal Depression Scale. Br J Psychiatry 1987, 150:782-786.

21. Braun V, Clarke V: Using thematic analysis in psychology. Qual Res Psychol 2006 3(2):77-101.

22. Stroebe $M$, Schut $H$ : The dual process model of coping with bereavement: rationale and description. Death Stud 1999, 23(3):197-224.

23. Ogwulu CB, Jackson LJ, Heazell AE, Roberts TE: Exploring the intangible economic costs of stillbirth. BMC pregnancy and childbirth 2015, 15:188. 
24. Nicholls J, Lawlor E, Neitzert E, T. G: A guide to social return on investment. In., 2nd edition edn. London: The Cabinet Office; 2012.

25. Heazell AE, Whitworth MK, Whitcombe J, Glover SW, Bevan C, Brewin J, Calderwood C, Canter A, Jessop F, Johnson $G$ et al: Research priorities for stillbirth: process overview and results from UK Stillbirth Priority Setting Partnership. Ultrasound Obstet Gynecol 2015, 46(6):641-647.

26. Hughes PM, Turton P, Evans CD: Stillbirth as risk factor for depression and anxiety in the subsequent pregnancy: cohort study. BMJ (Clinical research ed 1999, 318(7200):1721-1724.

27. DeBackere KJ, Hill PD, Kavanaugh KL: The parental experience of pregnancy after perinatal loss. $J$ Obstet Gynecol Neonatal Nurs 2008, 37(5):525-537.

28. Kim MY, Kim GU, Son HK: Hair Cortisol Concentrations as a Biological Marker of Maternal Prenatal Stress: A Systematic Review. Int J Environ Res Public Health 2020, 17(11).

29. Mustonen P, Karlsson L, Kataja EL, Scheinin NM, Kortesluoma S, Coimbra B, Rodrigues AJ, Sousa N, Karlsson H: Maternal prenatal hair cortisol is associated with prenatal depressive symptom trajectories. Psychoneuroendocrinology 2019, 109:104383.

30. Duffy AR, Schminkey DL, Groer MW, Shelton M, Dutra S: Comparison of Hair Cortisol Levels and Perceived Stress in Mothers Who Deliver at Preterm and Term. Biol Res Nurs 2018, 20(3):292-299.

31. Hughes $P$, Turton P, Hopper E, McGauley GA, Fonagy P: Disorganised attachment behaviour among infants born subsequent to stillbirth. J Child Psychol Psychiatry 2001, 42(6):791-801.

32. Wijngaards-de Meij L, Stroebe M, Schut H, Stroebe W, van den Bout J, van der Heijden PG, Dijkstra I: Parents grieving the loss of their child: interdependence in coping. Br J Clin Psycho/2008, 47(Pt 1):31-42.

33. Mistry $H$, Heazell $A E$, Vincent $O$, Roberts $T$ : A structured review and exploration of the healthcare costs associated with stillbirth and a subsequent pregnancy in England and Wales. BMC pregnancy and childbirth 2013, 13:236.

34. Hutti MH, Armstrong DS, Myers $\mathrm{J}$ : Healthcare utilization in the pregnancy following a perinatal loss. MCN Am J Matern Child Nurs 2011, 36(2):104-111.

35. Banke-Thomas AO, Madaj B, Charles A, van den Broek N: Social Return on Investment (SROI) methodology to account for value for money of public health interventions: a systematic review. BMC Public Health 2015, 15:582.

36. Wojcieszek AM, Heazell AE, Middleton P, Ellwood D, Silver RM, Flenady V: Research priorities and potential methodologies to inform care in subsequent pregnancies following stillbirth: a web-based survey of healthcare professionals, researchers and advocates. BMJ open 2019, 9(6):e028735.

\section{Figures}




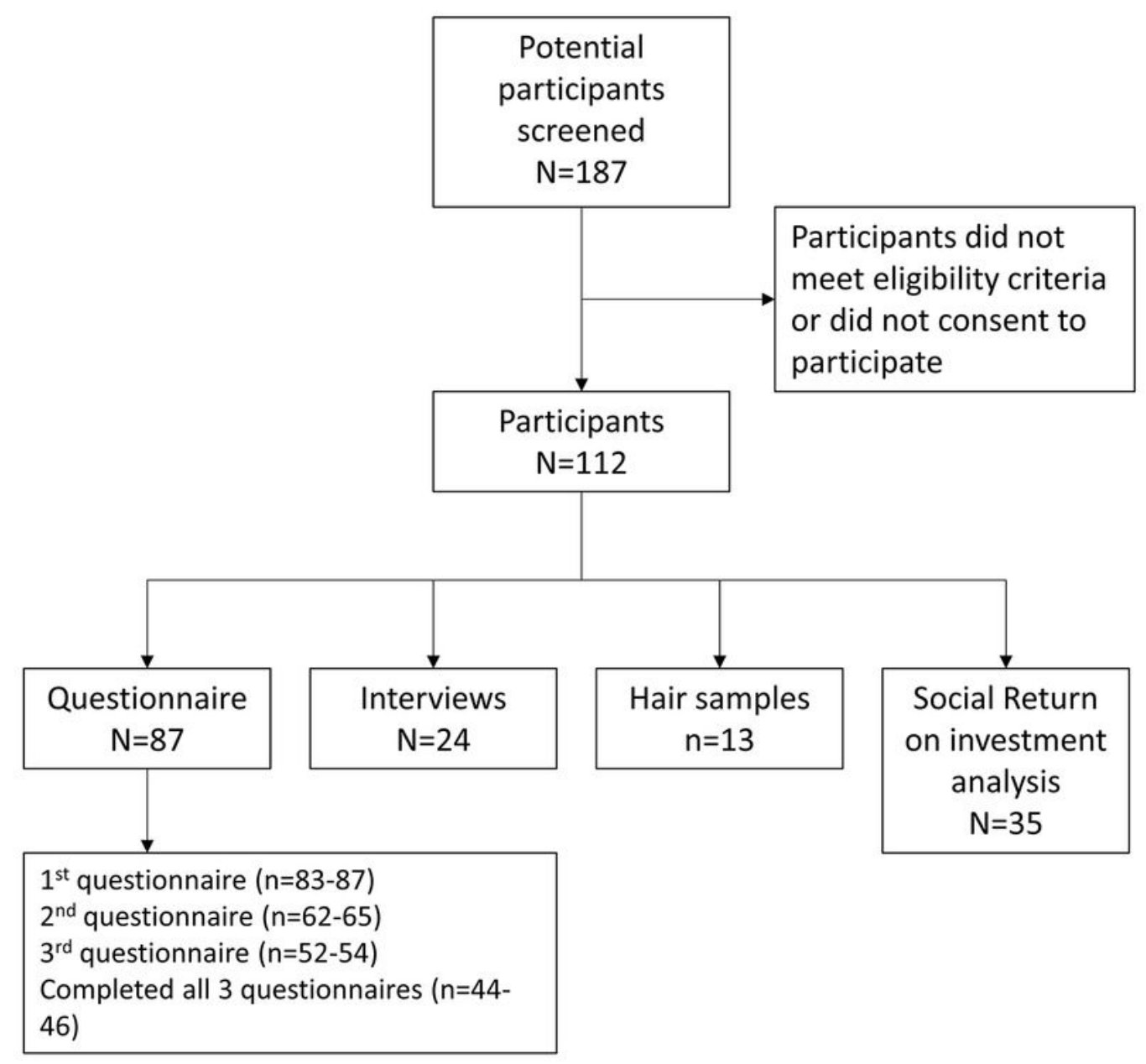

\section{Figure 1}

Flow chart indicating the number of women screened and the number of participants in different elements of the study. The total number of participants for individual components is greater than 112 because some women participated in more than one part of the study e.g. questionnaire and hair sample. 

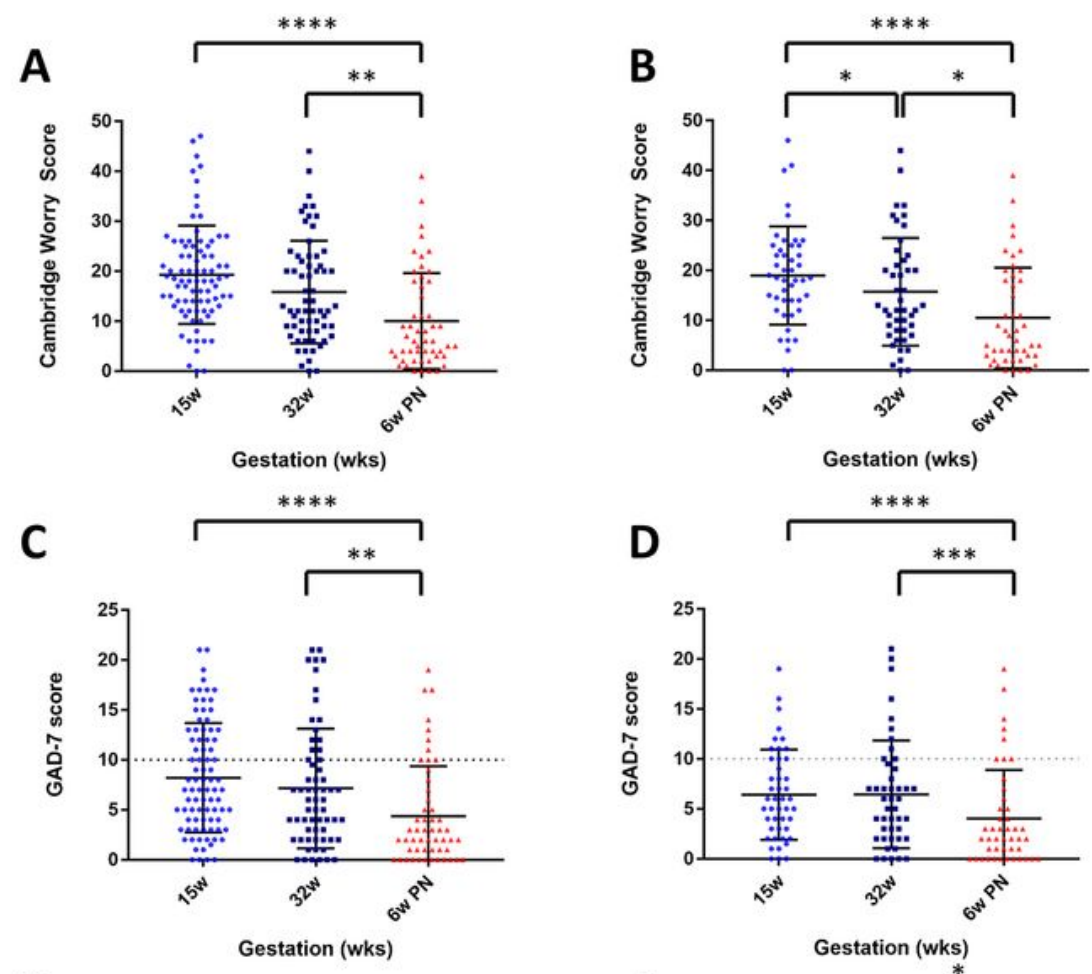

$\mathbf{E}$
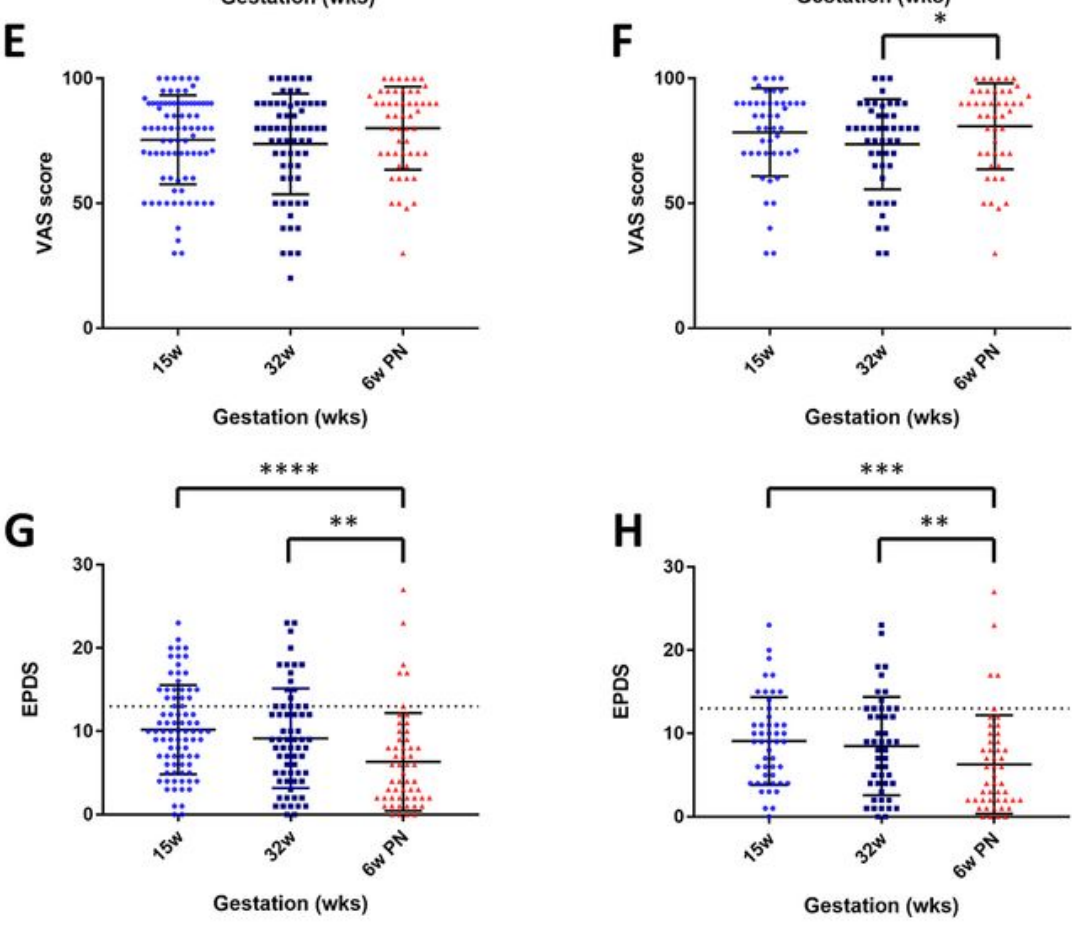

Figure 2

Results from psychometric and quality of life questionnaires. A) Cambridge Worry Score completed at 15 weeks' gestation, 32 weeks' gestation and 6 weeks postnatal for all participants, B) Cambridge Worry Score completed at the same gestations for participants who completed questionnaires at all three time points, C) GAD-7 completed at 15 weeks' gestation, 32 weeks' gestation and 6 weeks postnatal for all participants, D) completed at the same gestations for participants who completed questionnaires at all 
three time points, E) Visual Analogue Scale for quality of life (VAS) completed at 15 weeks' gestation, 32 weeks' gestation and 6 weeks postnatal for all participants, F) VAS completed at the same gestations for participants who completed questionnaires at all three time points, G) EPDS completed at 15 weeks' gestation, 32 weeks' gestation and 6 weeks postnatal for all participants, $\mathrm{H}$ ) EPDS completed at the same

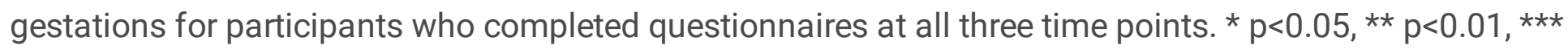
$\mathrm{p}<0.005, * \star \star \star \mathrm{p}<0.001$.

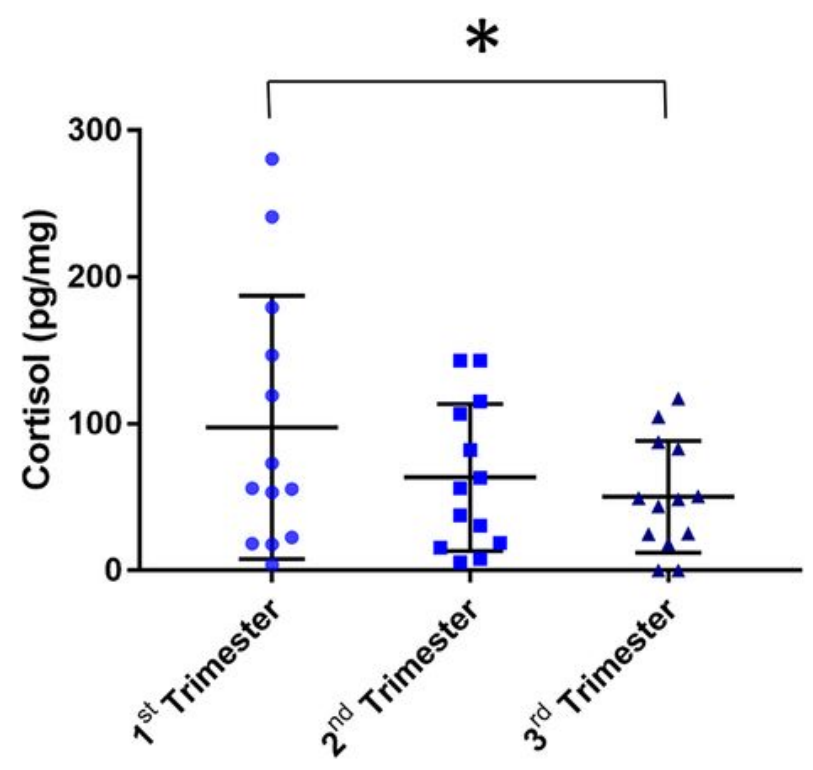

Figure 3 
Hair cortisol measurement in $\mathrm{pg} / \mathrm{ml}$ demonstrating a reduction in hair cortisol levels from the $1 \mathrm{st}$ through to the third trimester. ${ }^{*} p<0.05$.

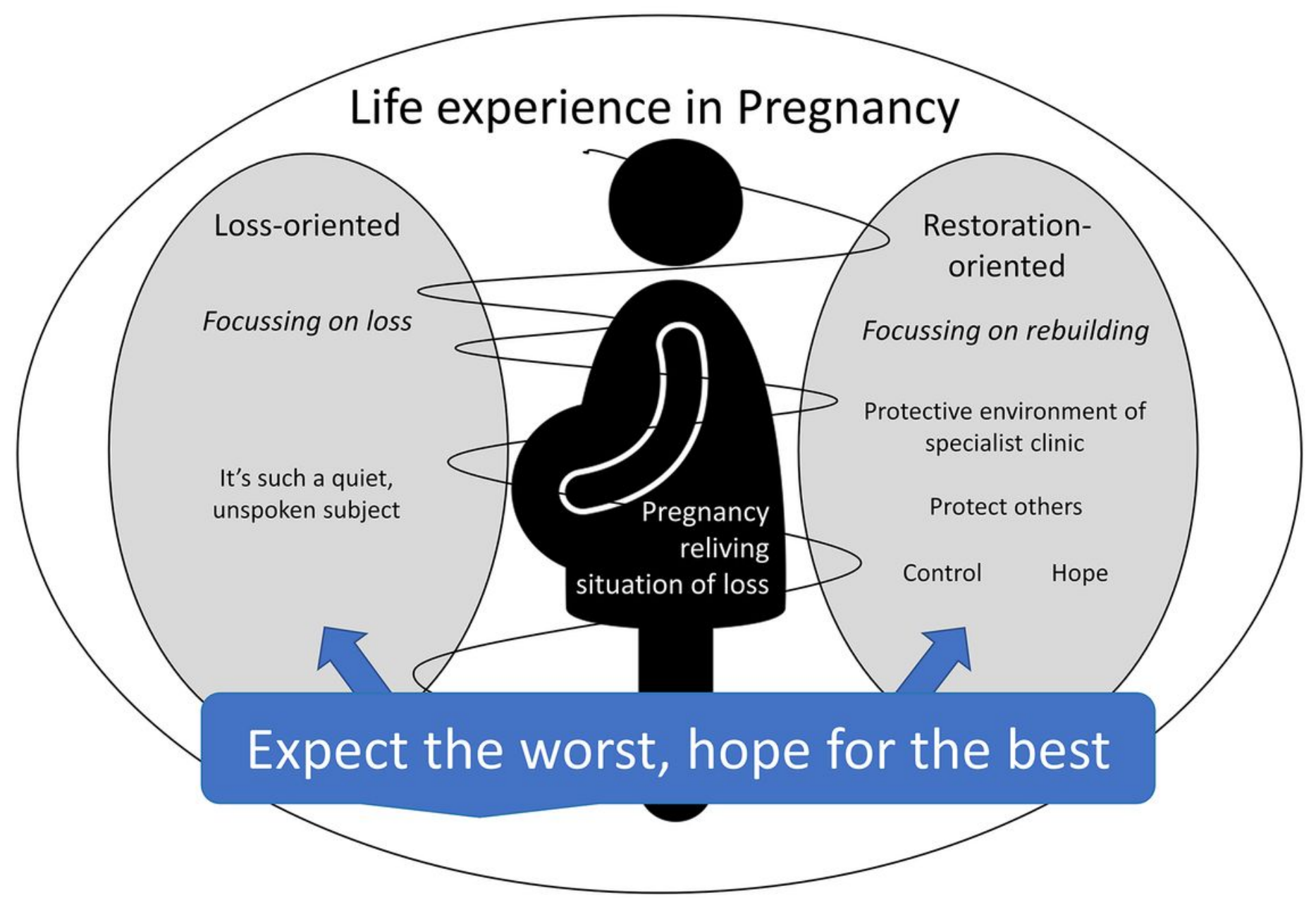

Figure 4

Schematic diagram mapping life experiences described in pregnancy on to the Dual Process Model of Grief [23]. Themes identified in semi-structured interviews are shown in loss-oriented or restoration oriented domains. 


\section{Image not available with this version}

\section{Figure 5}

Results of social return on investment analysis showing A) the proportion of change in a given domain attributed to attending the specialist antenatal service and B) the distribution of value generated across different stakeholders and outcomes. 